\title{
Evaluación diagnóstica sobre el conocimiento y viabilidad de uso del preservativo femenino: un estudio cualitativo
}

\author{
María Lameiras Fernández, M. a Victoria Carrera Fernández, Yolanda Rodríguez Castro, \\ M. ${ }^{a}$ Carmen Ricoy Lorenzo, José M. Failde Garrido y Ana M. Núñez Magana \\ Universidad de Vigo
}

\section{RESUMEN}

En esta investigación se presenta una evaluación diagnóstica a partir de las creencias, conocimiento, accesibilidad, actitud y viabilidad del uso del preservativo femenino, como punto de partida para el diseño e implementación de programas de promoción del método. Se desarrolla a través de un estudio de caso, en el contexto gallego, con un enfoque cualitativo y con la participación de 9 grupos de discusión, formados por un total de 83 jóvenes y adultos de edades comprendidas entre 19 y 42 años.

Entre los resultados más destacables identificamos que el preservativo femenino es poco conocido, escasamente utilizado y con una accesibilidad principalmente condicionada por su elevado precio e insuficiente difusión. Los agentes de educación formal e informal son los que promueven, de forma moderada, el acceso al método. Destacan actitudes neutras hacia el mismo.

La evaluación diagnóstica revela la necesidad de diseñar e implementar intervenciones educativas encaminadas a la promoción del uso del método.

Palabras clave: evaluación diagnóstica, preservativo femenino, intervención educativa, promoción de la salud, personas jóvenes y adultas, investigación cualitativa.

\section{ABSTRACT}

The present paper describes a diagnostic evaluation carried out to examine the beliefs, attitudes, and knowledge about, accessibility to, and acceptability of the female condom, information which can be used in designing and implementing interventional activities to promote this method. This is a qualitative case study using a sample of 83 adults (aged 19 to 42) from Galicia (Spain), divided into a total of nine discussion groups.

The results reveal that the female condom is not well-known, is hardly used, and has limited accessibility due to its price and a lack of adequate promotion. Probably, both informal and formal educational sources are responsible, to a moderate degree, for providing access to this method. It is also interesting to note that the attitudes held toward the female condom are neutral. The diagnostic evaluation carried out reveals a clear need to design and implement educational interventions aimed at promoting the female condom.

Keywords: Diagnostic evaluation, female condom, educational intervention, health education, young adults, qualitative research. 
Los estudios centrados en la difusión y la viabilidad de uso del preservativo femenino encuentran en el colectivo de mujeres adolescentes y jóvenes uno de los grupos diana de evaluación (Choi, Wokcicly, \& Valencia-García, 2004). Debido a los riesgos que supone una actividad sexual desprotegida en la exposición a infecciones de transmisión sexual -entre las que se encuentra la infección provocada por el Virus de la Inmunodeficiencia Humana (VIH)así como a embarazos no deseados (Robinson et al., 2002).

A nivel mundial, con más de 65 millones de personas infectadas por el VIH, la principal vía de contagio ha sido y continúa siendo la relación heterosexual desprotegida (Onusida, 2007). El informe de Desarrollo Humano de las Naciones Unidas identifica la pandemia del sida como el acontecimiento que supone el mayor retroceso en la historia del progreso humano (Programa de Naciones Unidas para el Desarrollo [PNUD], 2006). En España, con más de 70.000 casos de personas con sida, la transmisión heterosexual es la principal vía de contagio del VIH para las mujeres, adelantando a la vía sanguínea, a través de la que se infectaban, en su mayoría, consumidoras de drogas por el intercambio de instrumentos para el consumo intravenoso, así como la principal vía en incremento en los hombres (Instituto de Salud Carlos III, 2005). Y aunque en los últimos años se ha producido una estabilización en el volumen de nuevas infecciones, los datos de otras infecciones de transmisión sexual confirman la tendencia al alza. Por otro lado, el número de embarazos no deseados también se incrementa. En España, el informe realizado por el Instituto de la Juventud en 2005 constata que el 9,9\% de chicas menores de 21 años que ha tenido relaciones sexuales se ha quedado embarazada sin desearlo, de éstas el 31,3\% decidió abortar (Instituto de la Juventud [INJUVE], 2005).

En este contexto, el preservativo femenino representa el único método que puede usar la mujer, que ha demostrado tanto su eficacia contraceptiva (Far, Gabelnick, Sturgen, \& Dorfliger, 1994) como en la prevención de infecciones de transmisión sexual (Hoke et al., 2007). Además, los estudios confirman que la presencia del preservativo femenino incrementa el uso de otros métodos de barrera (Barbosa, Kalckmann, Berquó, \& Stein, 2007).

Los estudios sobre este nuevo recurso preventivo, disponible en el mercado desde 1994, se han centrado, por un lado en determinar su efectividad y por otro en evaluar su viabilidad de uso. Las investigaciones diseñadas para evaluar la seguridad-efectividad del preservativo femenino confirman su utilidad como método preventivo y anticonceptivo vinculado a un uso correcto y sistemático del producto (Farr, Gebelnick, Sturgen, \& Dorglinger, 1994). Por otro lado, los estudios encaminados a evaluar la viabilidad de uso se han centrado en determinar los conocimientos sobre el producto, las actitudes hacia el producto y los comportamientos de uso (Neilands \& Choi, 2002).

Además, las investigaciones disponibles, mayoritariamente desarrolladas en la última década, se han abordado en torno a dos grandes ejes: diferentes colectivos y diferentes lugares geográficos. En relación a los distintos colectivos evaluados, en su mayoría los estudios se han volcado en la viabilidad de uso en 
mujeres, especialmente en adolescentes y jóvenes, y en las conductas de riesgo que pueden incrementar su vulnerabilidad a infectarse de VIH o a un embarazo no deseado.

En cuanto a los diferentes lugares geográficos, la mayoría de las investigaciones se han circunscrito a países con altas tasas de incidencia del VIH. (Adeokun et al., 2002; Agha \& Van Rossem, 2002) y muy pocos de ellos han recurrido a muestras occidentales. De modo que se disponen de pocos estudios con muestras occidentales, en su mayoría realizados con mujeres norteamericanas (Lawson et al., 2003), siendo muy escasos los llevados a cabo en Europa (Gollub, Bros, Sovouillan Waterlot, \& Coruble, 2002; Spizzichino et al., 2007), y menos aún en nuestro país (Lameiras, Saco, Failde, \& Rodríguez, 2006; Núñez, 2006).

Las investigaciones llevadas a cabo confirman la mayor difusión en los últimos años del preservativo femenino, pero el uso continúa siendo escaso entre el colectivo de mujeres jóvenes en países occidentales (Haignere et al., 2000; Marshall, Giblin, Simpson, \& Backos, 2002; Okunlola, Morhason-Bello, Owonikoko, \& Adekunle, 2006). En esta línea, todavía se desconoce cómo y de que forma intervienen e interactúan las diversas variables que interfieren frente a las que favorecen la viabilidad de uso del preservativo femenino en mujeres jóvenes y adultas en nuestro país, así como en sus parejas heterosexuales.

Esta investigación se centra en la evaluación de las creencias y conocimientos de un grupo de personas jóvenes y adultas, así como de la accesibilidad, actitud y viabilidad de uso percibida sobre el preservativo femenino. Como punto de partida para el diseño e implementación de un programa de intervención educativa orientado a la promoción de uso del método en parejas heterosexuales estables. En este sentido, el estudio representa una primera aproximación en España al estudio de la promoción de uso del preservativo femenino a través de una metodología cualitativa.

\section{Método}

Con este trabajo se lleva a cabo una evaluación diagnóstica en su modalidad descriptivocomprensiva (Pérez, 2006), con el objeto de indagar sobre la temática ya referida. Asimismo, se buscan criterios racionales y científicos que permitan tomar decisiones sobre la planificación y desarrollo de programas de intervención educativa para promocionar el uso del preservativo femenino (Cohen \& Franco, 1993).

Esta investigación se realizó a través de un estudio de caso, desde el análisis de una unidad contextual mediante la técnica del grupo de discusión, en la comunidad autónoma gallega. Se trata del estudio de un caso particular, dado que su finalidad es el análisis y comprensión de una singularidad más que la generalización (Denzin \& Lincoln, 2000; Mcniff, Lomax, \& Whitehead, 2001). De forma que este trabajo se pone a disposición de la comunidad científica para que el lector/a pueda transferir sus aportaciones, desde un examen crítico, a otros contextos y grupos (Brown \& Yule, 1998, Feliz \& Ricoy, 2003).

\section{Participantes}

La selección de las personas que participan en el estudio se lleva a cabo mediante la invitación a colaborar y su aceptación opcional 
entre la población de estudiantes de diplomaturas y licenciaturas de la Universidad de Vigo (Campus de Vigo, Pontevedra y Ourense). Se han implicado en el estudio un total de 9 grupos de discusión, compuestos por 45 parejas, aunque en siete de ellas participó solamente uno de sus miembros. De estas personas 45 son mujeres y 38 varones, con un total de 83 sujetos con edades comprendidas entre los 19 y los 42 años.

La distribución de los grupos de discusión fue la siguiente: tres grupos de mujeres ( $\mathrm{G} 1, \mathrm{G}$ 4 y G 8, formados por 10 personas respectivamente); tres de varones ( 2 y $G$ 5, ambos con 6 varones; y $\mathrm{G} 9$ compuesto por 11 varones); dos mixtos ( $\mathrm{G} 3$ y $\mathrm{G}$ 6, conformados con 5 mujeres y 5 varones); y un grupo mixto de parejas (G 7 , con un total de 5 mujeres y sus respectivas parejas). La cantidad de grupos implicados se dejó inicialmente abierta para determinar su suficiencia atendiendo a la saturación en la información obtenida (Marcelo, 1992).

\section{Material}

El protocolo de las cuestiones, lanzadas a los grupos de discusión, se diseñó ad hoc para abordar el estudio planteado y se conformó con preguntas clave de carácter semiestructurado. Con la utilización del guión de preguntas se garantizó la uniformidad en la información recogida (Pelto \& Pelto, 1978). Por ello, los interrogantes planteados fueron los mismos para todos los/as participantes. Con estas preguntas se indaga en las creencias y conocimientos de las personas jóvenes y adultas sobre el preservativo femenino, así como sobre su viabilidad de uso.
La recogida de información se llevó a cabo a lo largo de 3 meses, durante el curso académico 2004/05. La discusión en los diferentes grupos se prolongó aproximadamente $40 \mathrm{mi}$ nutos. La recogida de datos fue llevada a cabo por un grupo de estudiantes de doctorado, que fueron formados para este cometido por los investigadores/as responsables.

\section{Análisis de la información}

Para realizar el análisis de contenido de los grupos de discusión hemos utilizado el programa AQUAD (Analysis of Qualitative Data). Este análisis de contenido de la información, obtenida con las preguntas planteadas a los grupos de discusión, se ha focalizado en las declaraciones, creencias, razonamientos, opiniones y valoraciones que relatan los participantes (Walker, 1989), con el objetivo de efectuar una interpretación coherente y respetuosa con la información que ha aflorado del debate.

El análisis de contenido desarrollado a partir de la discusión de los grupos se realizó a través de la grabación en audio y posterior trascripción literal de la información. La categorización primaria del análisis fue extraída de las preguntas planteadas a los grupos de discusión, mientras que las categorías específicas se derivaron de una concepción naturalista que aflora de la información obtenida (Goetz \& LeCompte, 1998). El proceso de categorización específica fue consensuado entre las/os investigadoras/es responsables.

Los análisis se realizaron a partir de la transcripción literal, en formato RTF, de la discusión mantenida en los grupos, profundizando en 
su contenido con el programa AQUAD (Analysis of Qualitative Data). Asimismo, el recuento de porcentajes y figuras se elaboró con la hoja de cálculo de Excel. Estos recuentos, emergen de la existencia de los datos producidos en la discusión de los grupos, originando las respectivas categorías específicas.

A través del programa AQUAD se captan diferentes núcleos de significado en la discusión, de los que se aportan algunos fragmentos ilustrativos en el apartado de resultados. De este modo, se presentan evidencias sobre la confluencia y singularidad de la información recogida a través del debate establecido en los grupos de discusión, asimismo se proporcionan pruebas al lector/a que le posibilitan la reflexión y el análisis. El recuento de categorías específicas que se ofrece permite el conocimiento sobre su predominio o excepcionalidad para profundizar en el análisis.

\section{Resultados}

Creencias y conocimiento sobre la existencia del método

Los resultados ponen de manifiesto que el preservativo femenino, a pesar de llevar en el mercado en torno a 15 años, es un método muy poco conocido entre las personas jóvenes y adultas sexualmente activas que han participado en el estudio (ver Figura 1). Asimismo, se observa que las creencias y conocimientos que poseen sobre el método son escasas, tal como puede percibirse en algunas de las manifestaciones expresadas en los siguientes fragmentos:

"Yo creo que la mitad de la gente no sabe que existe este preservativo» (G $6_{\text {[G.mixto]' }}$ mujer, línea 43); "Yo creo que la gente solo piensa en el masculino, no en este, no sabe ni que existe» (G.6 [G.mixto] mujer, línea 45-46); «Existe mucho desconocimiento sobre el tema, porque a la hora de la verdad se conoce la existencia, pero

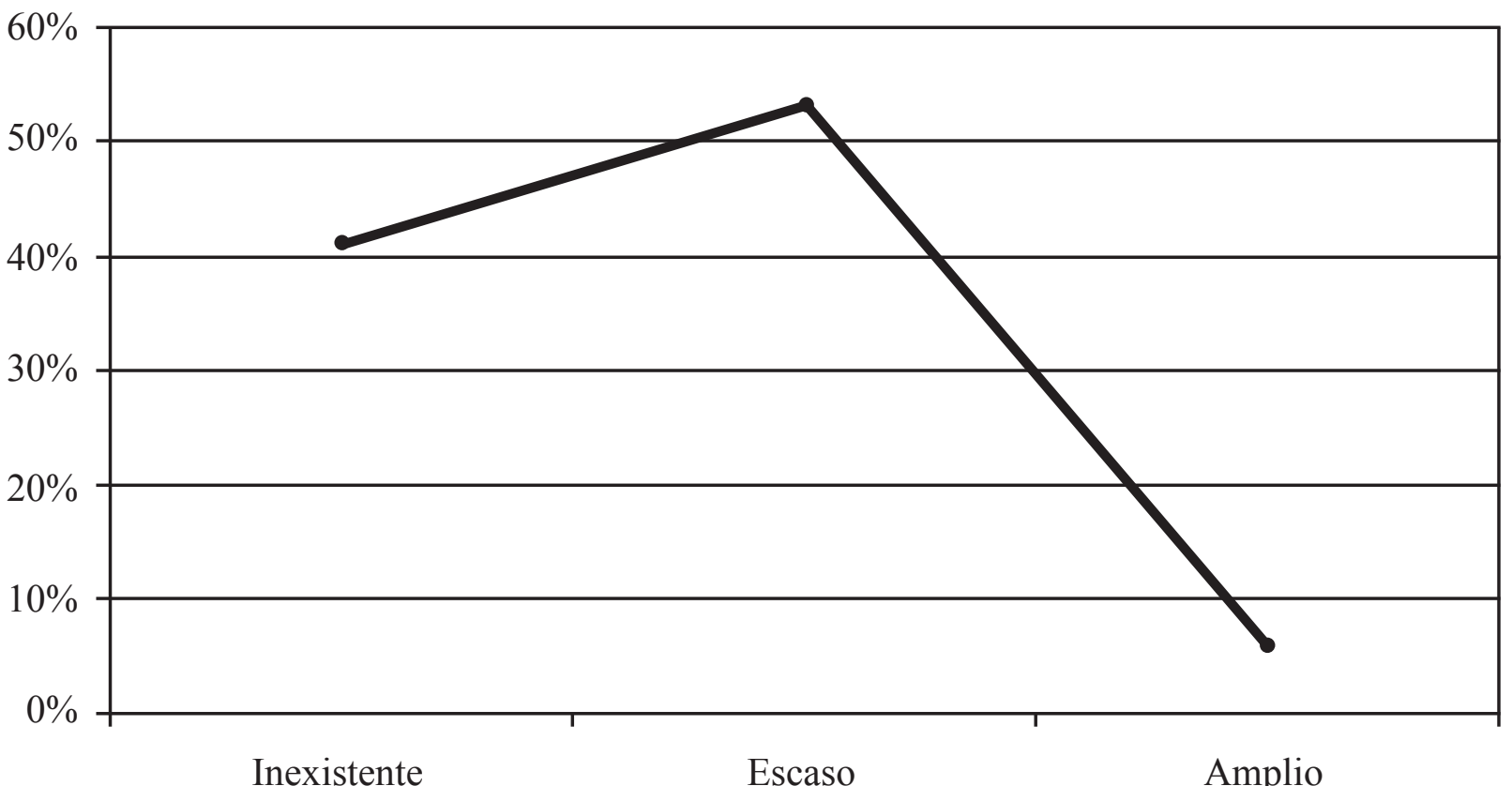

Figura 1. Creencias y conocimiento sobre el método. 
no se sabe mucho de ellos» (G $2_{\text {[G.hombres]' }}$ hombre, líneas 45-47).

Frente a la deficiente información, algunos grupos ponen de relieve que disponen de amplio conocimiento sobre la existencia del preservativo femenino. Dos de las chicas participantes suponen una excepción frente al desconocimiento que observamos en la mayoría de los grupos de discusión del estudio. Se trata concretamente de dos jóvenes que estaban realizando un trabajo en su facultad sobre el preservativo femenino, lo que, sin duda, explica su amplio conocimiento sobre el método: «Prácticamente lo conocemos todo... o, por lo menos, yo sí» (G $1_{\text {[G.mujeres]' }}$ mujer, línea 11).

Creencias y conocimiento sobre la accesibilidad al método

Otras creencias y conocimientos sobre el preservativo femenino giran en torno a su ac- ceso: precio, difusión y distribución (ver Figura 2). En este sentido, los/as participantes que se refieren al precio del producto señalan que es un método caro, tal y como podemos apreciar en los siguientes fragmentos:

"Sabía que era muy caro, yo había oído que valía en torno a 10 euros. Supongo que en unos años bajó» ( $2_{\text {[G.hombres] }}$, hombre, líneas 33-35); "Son carísimos, el precio que tiene debía ser el de una caja. Al cabo del año es peor que el tabaco» (G 2 [G.hombres]' hombre, líneas 195-196); "Una persona normal no se puede permitir usar cuatro de esos a la semana... ¿entiendes?... es imposible. Son carísimos» (G 4 [G.mujeres]' $_{\text {mujer, }}$ líneas 215-216).

En relación al excesivo precio del método son constantes las comparaciones que hacen los/as participantes con el preservativo masculino, utilizado como referente:

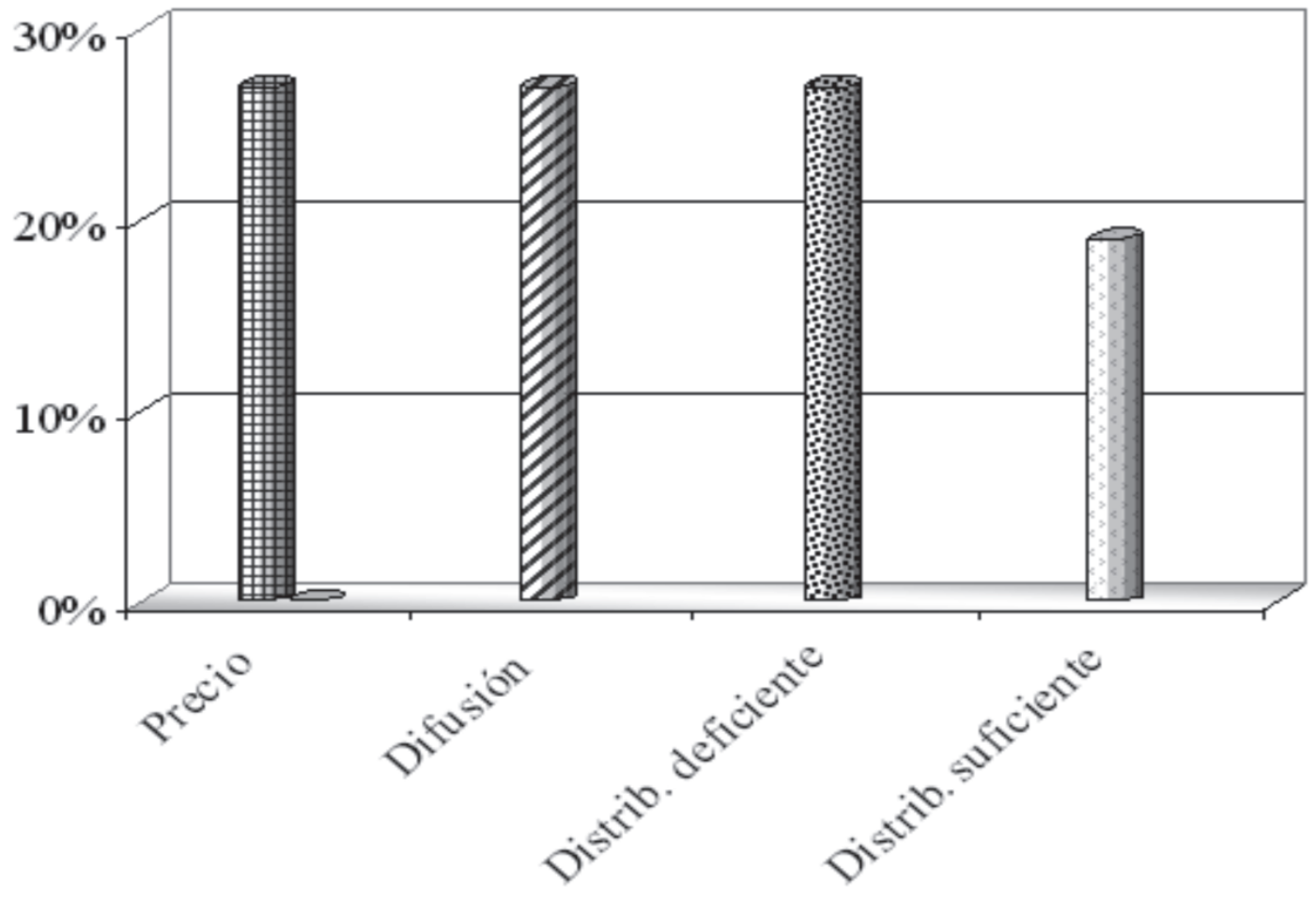

Figura 2. Elementos que condicionan la accesibilidad al método. 


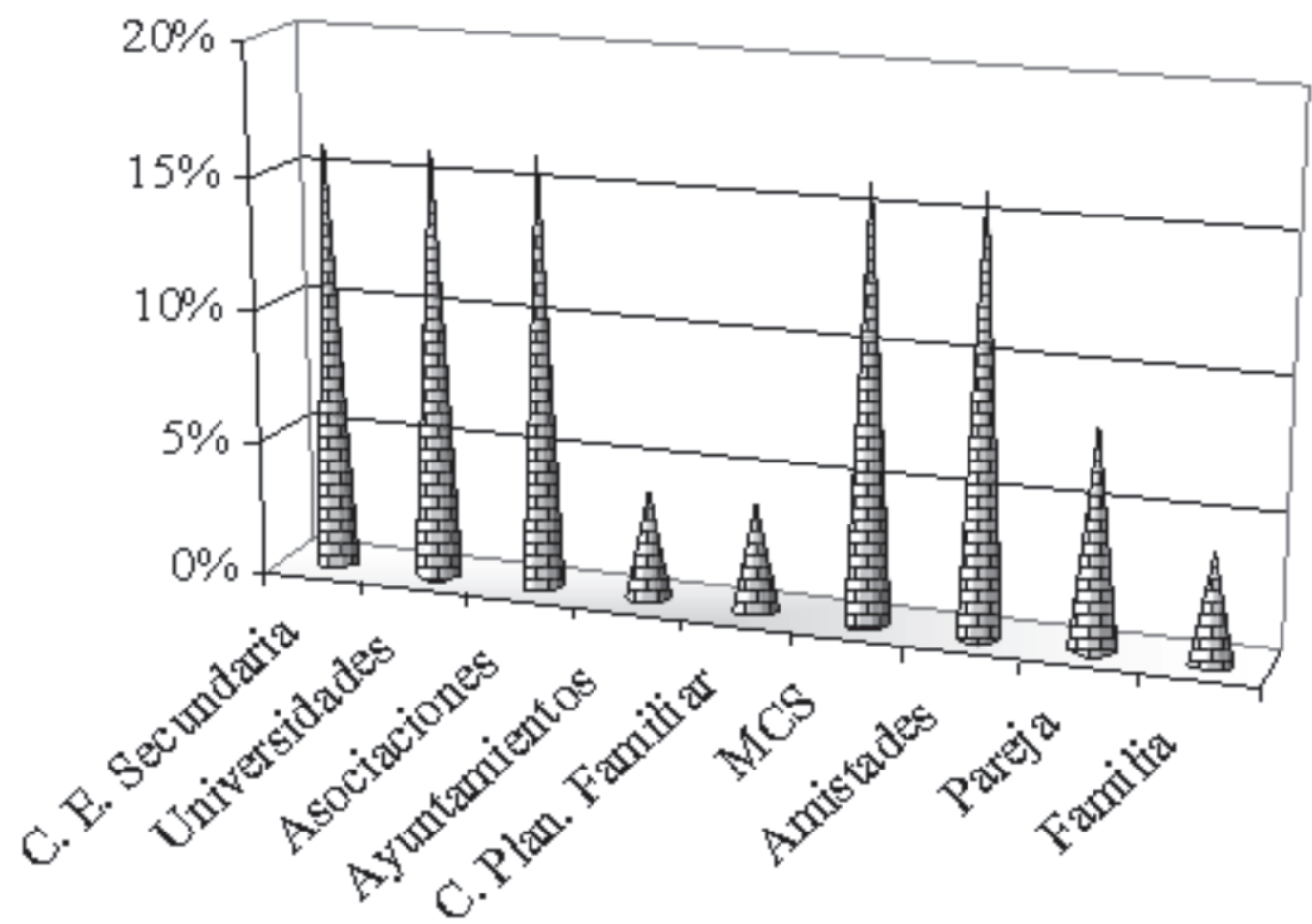

Figura 3. Medios de acceso al método.

«Hombre, es caro porque comparativamente con el masculino hay una diferencia importante» (G $2_{\text {[G.hombres] }}$ hombre, líneas 197-198); «Su precio es excesivo, comparado con el masculino» (G $4_{[\text {[G.mujeres] }}$ mujer, línea 192).

Sobre la difusión del preservativo femenino los/as participantes ponen de manifiesto que la promoción que se hace del producto es escasa, así como su divulgación: «Si nos fijamos en las campañas contra el sida... son todas con el preservativo masculino» (G 1 [G.mujeres] líneas 98-101); "La difusión del preservativo femenino es inexistente» ( $G 3_{\text {[G.mixto] }}$ hombre, línea 11); «En planificación familiar me hablaron del preservativo masculino y después de otros métodos como el parche y la píldora pero el preservativo femenino nunca me lo nombraron, incluso al hacer trabajos para el curso de monitores pedí información y nunca me lo nombraron. Te hablan de la píldora del día después y no te hablan de eso» (G $4_{\text {[G.mujeres]' }}$ mujer, líneas 179-184); «Yo no entiendo porque no se difunde más, si es porque no gusta o si hay gente que no está interesada en hacer pasta con él» (G 5 [G.hombres]' hombre, líneas 33-35).

En cuanto a la distribución del método, la mayor parte de los participantes sostienen que ésta es claramente deficiente, surgiendo de nuevo las comparaciones con el preservativo masculino como método de referencia:

"Yo fui el otro día a una farmacia a preguntar y me dijeron que fuera a planificación familiar» (G 1 [G.mujeres] ${ }$ mujer, líneas 355-356); «Es que en las farmacias tampoco te los ofrecen, yo iba a pedir unos preservativos de poliuretano y no me ofrecieron los femeninos, si los tienen y los conocen lo lógico es que los ofrezcan» (G $4_{\text {[G.mujeres] }}$ mujer, líneas 153-156); "Pero es que tú vas a una farmacia y le pides una caja de preservativos y te dicen ¿de doce o de seis?, no te preguntan ¿femenino o 
masculino?» (G $1_{\text {[G.mujeres]' }}$ mujer, líneas 341-343); «El acceso es más difícil que al masculino porque tu puedes encontrar máquinas expendedoras de masculinos en la calle o en los bares o gasolineras, pero de femeninos no, por lo tanto es mucho más fácil el acceso al masculino» (G $1_{\text {[G.mujeres] }}$ mujer, líneas 344-347); «Si no está la farmacia o el sexshop abierto entonces que haces, ya no lo puedes usar» (G $1_{\text {[G.mujeres] }}$ mujer, líneas 351-352); «En las farmacias, nadie te pregunta qué preservativo quieres masculino o femenino, lo dan por hecho... Y seguro que si eres tío la cosa esta clara, lo que me pregunto es si eres tía si te lo preguntarían o no» (G $9_{\text {[G.hombres] }}$ hombre, líneas 68-72).

En este sentido, son pocos los/as participantes de los grupos de discusión que afirman que la distribución del método es aceptable. De modo que los escasos esfuerzos realizados hasta el momento en su difusión y distribución podrían estar influyendo en la reducida utilización del método y, como veremos, en las actitudes poco positivas manifestadas sobre el mismo. No obstante, se pueden aportar algunos ejemplos esperanzadores:

"Yo creo que en cualquier farmacia que se entre pidiéndolo te lo darán» ( $\mathrm{G} 2$ [G.hombres] ${ }^{\text {hom- }}$ bre, líneas 174-175); «Yo creo que no sería difícil encontrarlo» (G 5 [G.hombres] hombre, línea 137); «Bueno, yo creo que el acceso es parecido al masculino, porque sacando que por la noche no lo puedes encontrar en locales, pues del resto lo compras en farmacia, incluso en una farmacia de guardia» (G $1_{\text {[G.mujer] }}$ mujer, líneas 367-369).

Medios de acceso al método

Por lo que respecta al medio de acceso al método, los/as participantes destacan que el acceso al conocimiento del preservativo feme- nino se realiza a través de diferentes agentes educativos y de socialización, tanto de carácter formal (centros de educación reglada), como no formal (instituciones que posibilitan formación no reglada) e informal (medios de comunicación social, familia, pareja y amigos). Tal y como podemos observar en la Figura 3.

Por otra parte, se comprueba que los diferentes agentes de educación informal (medios de comunicación social y amistades), junto a los de educación formal (centros de educación secundaria y universidades) se encuentran entre los medios que principalmente promueven el conocimiento del preservativo femenino. En los siguientes fragmentos puede constatarse lo referido:

«Yo sólo había visto el preservativo femenino en un periódico» (G $4_{\text {[G.mujeres]' }}$ mujer, línea 11); «Yo había oído hablar sobre este método en un telediario» (G $4_{\text {[G.mujeres] }}$ mujer, líneas 12-13); "Yo conocía este preservativo, pero por la televisión, de escucharlo en la tele, en un programa de sexo, el de...» (G $8_{\text {[G.mujeres]' }}$ mujer, líneas 14-16).; “Yo no lo conocía, pero el año pasado lo empezó a utilizar una amiga mía porque tenía problemas con la píldora y con otros métodos anticonceptivos y me lo enseñó, pero... con el envoltorio puesto, sabes nunca tuve la oportunidad de tocarlo» (G 1 [G.mujeres]' mujer, líneas 34-37);

Además, tal y como se ha señalado anteriormente, la propia pareja y la familia intervienen, aunque de forma reducida, en la promoción de uso del preservativo femenino:

«A mí me habla de este método mi novio, me dijo: hay preservativos femeninos, y me parece que ya lo tenía oído, pero nada más, la primera fuente de información pienso que fue él» (G 1 


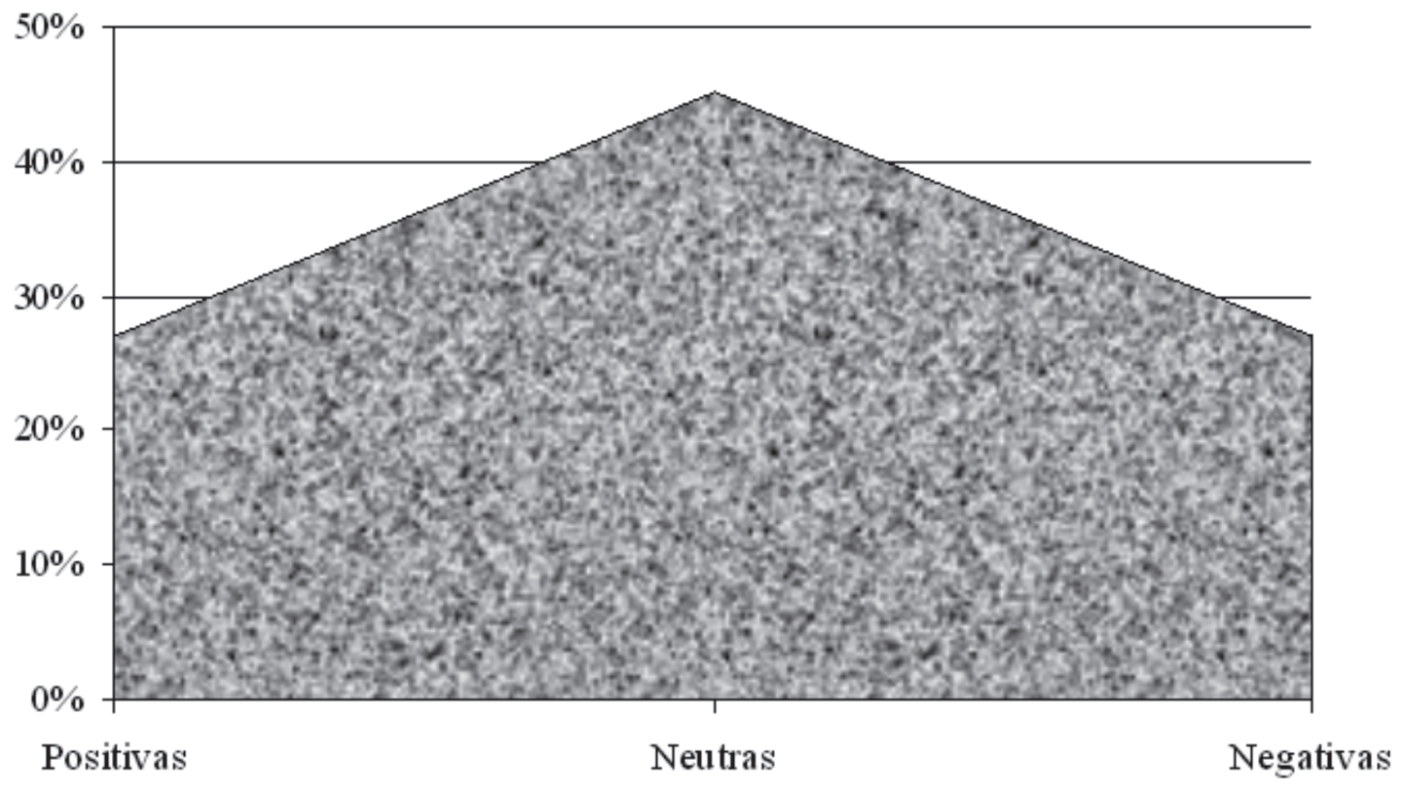

Figura 4. Actitudes hacia el método.

[G.mujeres]' mujer, líneas 45-47); «Lo conocía porque mi madre tuvo que usar todas las alternativas que hay hasta probar a ver qué método le iba mejor» (G 8 [G.mujeres]' mujer, líneas 29-32).

Otra de las vías de conocimiento del método identificadas han sido los agentes de educación formal, concretamente los centros de educación secundaria y las universidades; aunque esta difusión es escasa y debería incrementarse. Seguidamente ofrecemos algunos fragmentos ilustrativos:

"Yo no lo he tocado, pero si he tenido la típica charla del cole que te dan información sobre la sexualidad. Yo estaba haciendo segundo o tercero de BUP, nos venían a dar charlas para conocer los métodos anticonceptivos que había, pero no lo tocamos, lo he visto solamente» (G $1_{\text {[G.mujeres]' }}$ mujer, líneas 21-25); "Conocí el preservativo femenino a través de una charla, sobre planificación familiar, en el instituto» ( $G 6_{\text {[G.mixto] }}$ hombre, línea 10); "Yo cuando estaba haciendo Educa- ción Social, teníamos unos seminarios para escoger y había escogido el de educación sexual y nos habían hablado de todo esto y nos lo enseñaron» (G $4_{\text {[G.mujeres]' }}$ mujer, líneas, 18-20).

La difusión llevada a cabo en los centros de educación formal pone de manifiesto la precariedad con la que se aborda la educación sexual en nuestro contexto. Con todo, las instituciones educativas apuntan a la existencia de una educación sexual reduccionista, de carácter biologicista y reproductivo, aplicada de forma esporádica y no sistemática, a través de charlas y conferencias de personas normalmente ajenas a la institución educativa.

Junto a la difusión promovida por los agentes de socialización formal e informal, destacan los esfuerzos realizados por los centros de educación no formal, tales como asociaciones culturales, centros juveniles y ayuntamientos, como señalaron algunos de los participantes de los grupos de discusión: 


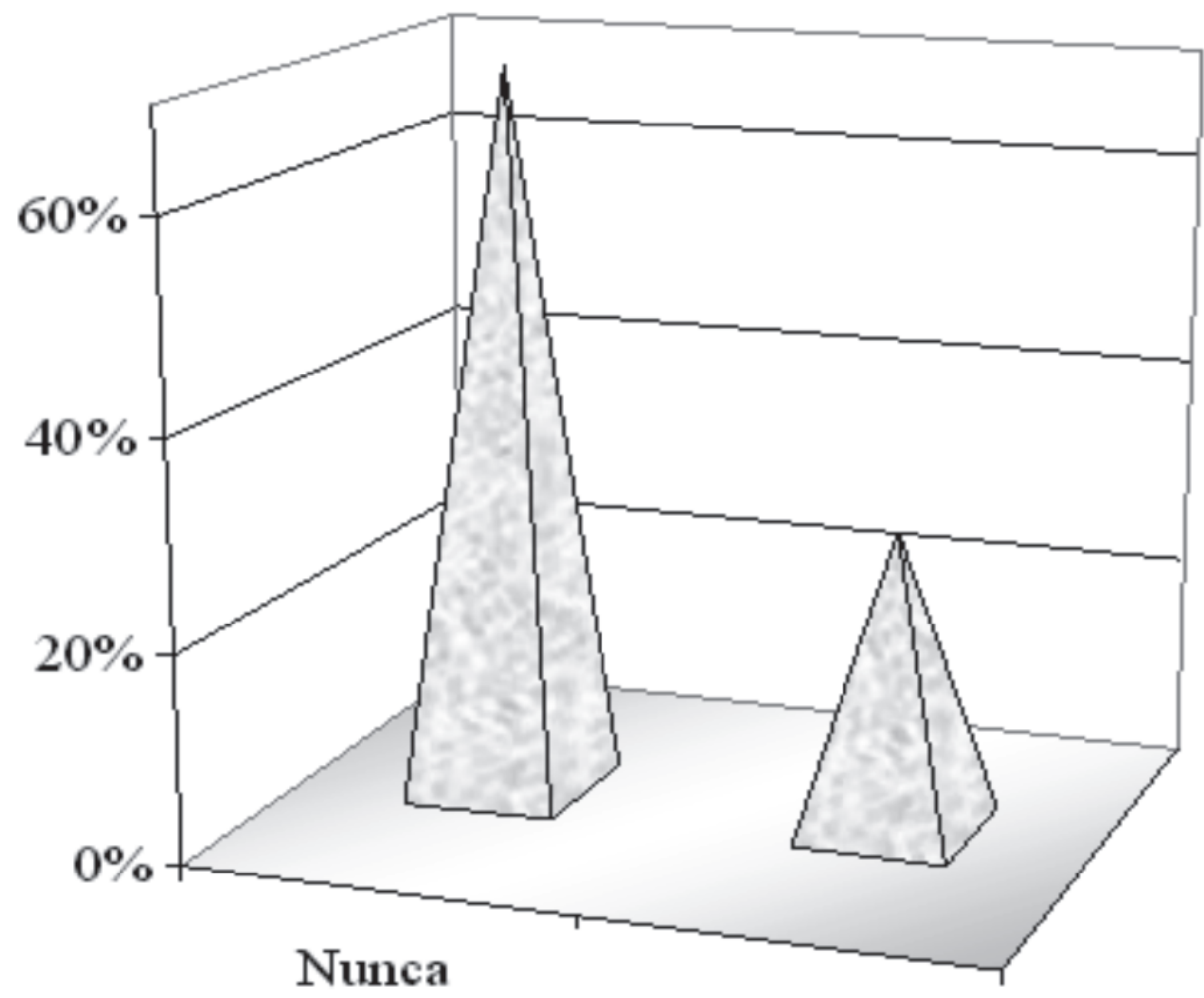

Alguna vez

Figura 5. Uso y viabilidad del método.

"Yo estudié en un colegio de monjas y no lo conocía pero de alguna información del ayuntamiento que hacen a través de campañas, tenía alguna idea aunque nunca lo había visto» (G 1 [G.mujeres]' mujer, líneas 31-33); «A mi el preservativo femenino me lo dieron los de Cruz Roja, pero no sabía lo que era» (G $3_{\text {[G.mixto] }}$ mujer, línea 30); «Yo conocí este método en un curso para monitores de tiempo libre, porque como le tenemos que enseñar también a adolescentes los métodos anticonceptivos yeso, pues... nos lo explicaron a nosotros también. Fue la única información que tuve» (G 4 [G.mujeres]' mujer, líneas, 7-10); «Yo me lo encontré en uno de esos folletos de la Asociación Alecrín, de repente ponía, preservativo femenino y yo flipé, ¡vale!» (G 7 [G.mixto parejas]' hombre, líneas 30-31).

Además, dentro de los centros de educación no formal, destaca la escasa difusión realizada por los centros de planificación familiar:

«Fui a un centro de planificación y allí me lo explicaron» (G $1_{\text {[G.mujeres] }}$ mujer, líneas 37-39); «Conozco el preservativo femenino a través de panfletos de planificación familiar, de recoger información sobre métodos anticonceptivos y de verlos en dibujos, pero nunca los toqué» (G 1 [G.mujeres]' mujer, líneas 40-42).

\section{Actitudes hacia el método}

Las actitudes hacia el preservativo femenino se han analizado a través de las declaraciones, descripciones, opiniones y valoraciones que manifiestan en el debate los/as participantes de los diferentes grupos de discusión. Identificándose actitudes positivas, negativas y neutras hacia el método (ver Figura 4). 


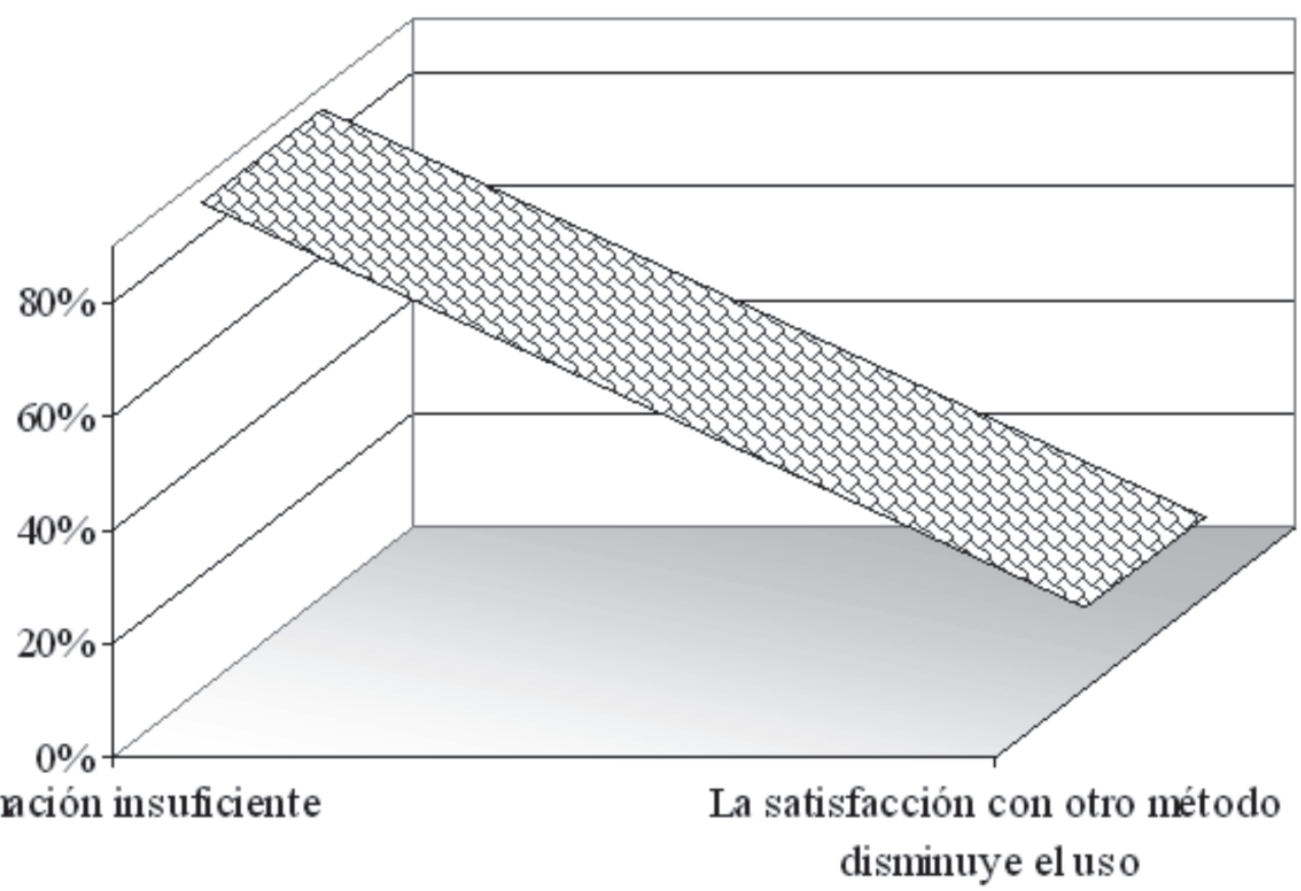

Figura 6. Viabilidad de uso del método.

Las descripciones que los participantes realizan sobre el preservativo femenino dejan entrever unas actitudes mayoritariamente neutras hacia el método, que ponen de relieve una imagen no preconcebida del mismo, así como descripciones que no traslucen actitudes positivas ni negativas: "Sabía que era parecido al masculino, pero para la mujer» (G $4_{\text {[G.mujeres]' }}$ mujer, línea 25).

Por otra parte, se encuentran también en una proporción equivalente descripciones que evidencian unas actitudes tanto positivas como negativas. Las actitudes positivas se manifiestan a través de la comparación o asimilación del preservativo femenino con otros métodos anticonceptivos ampliamente conocidos e interiorizados, fundamentalmente con su homólogo masculino, pero también con el diafragma, con el que a veces se confunde: «Pensé que era como el masculino, que no tenía esa forma. Fue lo que más me sorprendió: la for- ma pero no pasa nada» (G 5 [G.hombres]' hombre, líneas 8-9); "Yo pensé que era como el diafragma, de hecho creía que era lo mismo aunque hay diferencia» (G 8 [G.mujeres]' mujer, líneas 62-63).

Por su parte, las actitudes negativas hacia al método se manifiestan a través de adjetivos que hacen referencia a su gran tamaño y aparatosidad, $y$, en general, a una imagen poco atractiva y estética del método:

«Pensé que sólo tenía una aro, no dos y que era como... un colador» (G $1_{\text {[G.mujeres] }}$ mujer, línea 46); «Yo la información que tenía no era poca.... en cuanto me enteré que era de poliuretano dudé de él, porque había oído a una chica que era alérgica al látex y tenía que usar preservativos de poliuretano, que aquello era como ponerse una bolsa, y entonces sí que me causó algo de rechazo cuando vi que era poliuretano, entiendes, un poco de prejuicio quizá tengo» (G 2 [G.hombres]' hombre, líneas 81-87).

Las actitudes negativas que ponen de manifiesto el rechazo al método están relaciona- 
das con el escaso conocimiento que tienen del mismo, así como con la imagen preconcebida del método basada en la interiorización y asociación con el preservativo masculino.

\section{Uso y viabilidad del método}

Sobre la utilización y viabilidad de uso del preservativo femenino hay que destacar que la mayor parte de las parejas que han participado en los grupos de discusión señalan no haber utilizado nunca este método. Además, de éstas parejas destacan aquellas que especifican no haberse planteado nunca el uso del método. Frente a la mayor parte de los/ as participantes, sólo una pareja afirma haber utilizado el preservativo femenino en alguna ocasión.(ver Figura 5).

Como fragmentos ilustrativos pueden revisarse los siguientes:

«Ni lo había utilizado, ni lo había tocado, ni nada» (G 1 [G.mujeres]' mujer, líneas 50-51); "Más o menos lo mismo que todos, lo conocíamos, pero no lo habíamos probado» (G 2 [G.hombres]" hombre, líneas 43-44); «Yo lo vi en el instituto, y también en medios de comunicación, pero nunca lo usara» (G $4_{\text {[G.mujeres] }}$ mujer, líneas 27-28); «No me había planteado nunca utilizarlo» (G $1_{\text {[G.mujeres] }}$ mujer, líneas 25-26); "Yo ya lo utilicé por lo menos hace ocho años» (G 8 [G.mujeres]' mujer, línea 41); «Lo probamos hace más o menos 5 años» (G 9 [G.hombres]' $_{\text {, }}$ hombre, líneas 27-28).

En relación a la viabilidad de uso, los/as jóvenes y personas adultas señalan que la información de la que disponen es insuficiente para utilización del preservativo femenino, incidiendo en la desconfianza que genera un método preventivo del que conocen poco. Asimismo, una mujer pone de manifiesto que la satisfacción con el método utilizado disminuye la viabilidad de empleo del preservativo femenino (ver Figura 6).

Como evidencias sobre la viabilidad de uso del preservativo femenino aportamos los siguientes fragmentos:

«Al tener información sobre un método estás más dispuesta a usarlo porque lo ves desde otros ojos. Si la tienes incompleta dudas, no te fías de su capacidad de protección, entonces igual te haces más reacia para probarlo» (G 1 [G.mujeres]' mujer, líneas 69-75); «Yo creo que el preservativo femenino es el gran desconocido, no se conoce, nadie prueba porque no sabe ni si va a dar buen resultado, entonces vas a lo mejor, a lo que siempre te da resultado» (G 8 [G.mujeres] mujer, líneas 108-111); "Yo creo que la gente está cómoda con lo que tiene, toma la píldora o el preservativo masculino, $y$ tiene la información que quiere tener, porque te puedes informar por muchas partes si quieres. Yo estoy cómoda... y para qué saber nada más» ( $G$ $1_{\text {[G.mujeres]' }}$ mujer, líneas 94-97).

\section{Discusión}

En relación al conocimiento sobre la existencia del preservativo femenino, se ha descubierto que, a pesar del tiempo que lleva comercializándose, es un método desconocido entre las personas jóvenes y adultas. Ello, hasta el punto de que este vacío informativo del método es prácticamente generalizado.

Los estudios confirman que el preservativo femenino es un método escasamente conocido y poco utilizado tanto fuera (Haignere et al., 2000) como dentro de nuestras fronteras (Lameiras et al., 2006; Lameiras, Núñez, Rodríguez, Breton, \& Agudelo, 2007). 
Los/as participantes consideran que el precio del preservativo femenino es elevado y la difusión escasa, siempre en comparación con su homólogo masculino. Las referencias al elevado precio como una de las principales limitaciones para su uso son constantes en la literatura disponible (Welbourn, 2006). Así, a pesar de que el preservativo femenino es elaborado para un único uso, la fortaleza demostrada los hace aptos para su reutilización, con lo que se podría superar una de las principales barreras para su uso. Las investigaciones demuestran que estos preservativos pueden soportar un nivel de reutilización sin pérdida de la integridad estructural cuando se lavan adecuadamente con agua y jabón (Smith, Nkhama, \& Trottier, 2001).

En relación a la difusión, se pone de relieve la escasa promoción y divulgación del método. Por su parte, la distribución del preservativo femenino ha dado lugar a opiniones enfrentadas, aunque la mayor parte de los/as participantes sostiene que ésta es claramente deficiente, estableciendo de nuevo comparaciones con el preservativo masculino como método de referencia.

La disponibilidad de preservativos femeninos de bajo coste en farmacias o supermercados, así como su distribución gratuita por instituciones continúa siendo una tarea pendiente (Prudhomne, Boucher, Delberghe, Christiman, \& Leroux, 2005), que ha demostrado ser muy exitosa en la distribución del preservativo masculino. Indudablemente, los escasos esfuerzos de difusión y distribución influyen directamente en el precario conocimiento del método y en el desarrollo de actitudes poco favorables hacia el mismo.
Los diferentes agentes educativos y de socialización, que incluyen tanto los de educación formal y no formal, como aquellos que se sitúan en la dimensión de la educación informal (Trilla, 1993), sirven también como medios de acceso para el conocimiento del preservativo femenino. Siendo los agentes de educación informal, particularmente a través de los medios de comunicación y las amistades, los que contribuyen notablemente a la difusión del método. Las investigaciones confirman el papel prioritario que los medios de comunicación tienen en la difusión de información sobre el preservativo femenino (Okunlola et al., 2006).

Entre los agentes de educación formal, se aprecian el papel que desempeñan los centros de Educación Secundaria y la Universidad. En este sentido, las tareas de difusión llevadas a cabo por las instituciones de educación formal ponen de manifiesto la presencia de la educación sexual de carácter meramente reduccionista, biologicista y reproductivo, aplicada de forma puntual y no sistemática a través de charlas y conferencias de personas ajenas al centro (Amezúa \& Foucart, 2005; De la Cruz, 2003; ). No obstante, el presente estudio no aporta datos suficientes para conocer la verdadera naturaleza y alcance de la misma.

Asimismo, ejercen una labor interesante los centros de educación no formal, tales como ayuntamientos, asociaciones culturales y recreativas. Destacando la escasa difusión realizada por los centros de planificación familiar, especialmente orientados a esta tarea. En esta línea, las investigaciones encaminadas a analizar las vías de difusión del preservativo femenino destacan el papel que juega el personal 
que trabaja en estos centros, confirmándose la necesidad de reducir en estos colectivos las percepciones negativas que todavía subsisten sobre el preservativo femenino (Mantel, Hoffman, Exner, Stein, \& Atkins, 2003). En esta línea, crear una mayor conciencia y formación entre los/as profesionales sobre el preservativo femenino se considera prioritario no sólo en el ámbito sanitario sino en el educativo (Preusseler, Micheletti, \& Pedro, 2002).

En relación a las actitudes manifestadas hacia el método, analizadas a través de los comentarios relativos a su imagen, se denotan posicionamientos imparciales hacia el mismo. Así, las descripciones de los/as participantes dejan entrever unas actitudes neutras hacia el preservativo femenino, que pone de relieve una imagen no preconcebida sobre éste. Existen evidencias menos manifiestas sobre actitudes tanto positivas como negativas hacia el método. Las actitudes positivas se reflejan a través de la comparación o asimilación del preservativo femenino con otros métodos anticonceptivos ampliamente conocidos e interiorizados, fundamentalmente con el masculino, pero también con el diafragma, con el que existe cierta confusión. La comparación del preservativo femenino con su homólogo masculino es recurrente (Agha, 2001).

La actitud negativa hacia este método se plasma a través de adjetivos que hacen referencia a su gran tamaño y aparatosidad, y en general a una imagen poco atractiva y estética. Esta actitud de rechazo hacia el método está relacionada con el poco conocimiento que existe del mismo, así como con la presencia de una imagen preconcebida basada en la arraigada interiorización y asimilación del preservativo masculino, confrontación en la que resulta dañada la percepción que se tiene del preservativo femenino (Kulczycki, Kin, Duerr, Jamieson, \& Macaluso, 2004).

Sobre el uso y la viabilidad de uso de este método, se comprueba que prácticamente la totalidad de los/as participantes nunca lo ha utilizado. Destacan, además, las declaraciones de aquellos que especifican no haberse planteado nunca el uso del método. En relación a la viabilidad, se pone de manifiesto que la información disponible sobre el producto es insuficiente para tomar decisiones sobre la utilización del mismo y, también, se vislumbra la desconfianza que genera un método preventivo que se conoce poco. Por otra parte, algunos grupos sostienen también que la satisfacción obtenida con el método utilizado disminuye la viabilidad de uso del preservativo femenino. Sin embargo, ésta no ha de ser considerada como una limitación, ya que la promoción de uso del preservativo femenino ha de ir encaminada a permitir que éste sea incorporado como una alternativa más, no como disyuntiva a otros métodos anticonceptivos y sobre todo preventivos. De hecho, en recientes investigaciones se ha destacado la utilidad de promover conjuntamente ambos métodos preventivos (Mantel, Hoffman, Exner, Stein, \& Atkins, 2003). Por lo que, considerar el uso del preservativo femenino como complementario al masculino conduce a un aumento de las relaciones sexuales protegidas (Choi, Gregorich, Anderson, Grinstead, \& Gómez, 2003).

Sin embargo, las limitaciones sobre el uso del preservativo femenino que se desprenden de este estudio no deben desalentar. La his- 
toria del tampón demuestra que la viabilidad de uso de un método no viene condicionada por la aceptación inmediata por todos los/as potenciales usuarios/as, como lo fue éste en su momento, llegando a ser posteriormente ampliamente aceptados (Latka, 2001).

Finalmente, es necesario destacar que sólo el trabajo constante y conjunto de los/as profesionales de la educación y de la sanidad, así como el esfuerzo de las autoridades sociales y políticas, permitirán una amplia difusión y conocimiento del método, contribuyendo así a su promoción de uso.

\section{Referencias}

Adeokun, L., Mantell, J. E., Weiss, E., Delano, G., Jagha, T., Olatoregun, J., Udo, D. Akiso, S. \& Weiss, E. (2002). Promoting dual protection in family planning clinics in Ibadan, Nigeria, International Family Planning Perspectives, 28, 87-95.

Agha, S. (2001). Intention to use the female condom following a mass- marketing campaign in Lusaka. American Journal of Public Health, 91, 307-310.

Agha, S., \& Van Rossem, R. (2002). Impact of mass media campaigns on intentions to use the female condom in Tanzania. International Family Planning Perspectives, 28, 151-158.

Amezúa, E., \& Foucart, N. (2005). El libro de los sexos V: educación sexual. Guía para el profesorado. Primera parte: las bases teóricas y esquemas generales [Monografía]. Revista Española de Sexología, 127-128, 13-198.

Barbosa, R.M., Kalckmann, S., Berquó, E., \& Stein, Z. (2007). Notes on the female condom: experiences in Brazil. Internacional Journal of STD\&AIDS, 18, 261-266.
Brown, G., \& Yule, G. (1998). Discourse analysis. Cambridge: University Press.

Chen, M. P., Macaluso, M., Blackwell, R., Galvao, L., Kulczcki, A., Jamieson, D. J., \& Duerr, A. (2007). Self-reported mechanical problems during condom use and semen exposure. Comparison of two randomized trials in the United States of America and Brazil. Sexually Transmitted Diseases, 34, 557-562.

Choi, K. H., Gregorich, S. E., Anderson, K., Grinstead, O., \& Gómez, C. A. (2003). Patterns and predictors of female condom use among ethically diverse women attending family planning clinics. Sexually Transmitted Diseases, 30, 91-98.

Choi, K., Wojcicky, J., \& Valencia-García, D. (2004). Introducing and negotiating the use of female condoms in sexual relationships: Qualitative interviews with attending a family planning clinic. Aids and behavior, 8 , 251-266.

Cohen, E., \& Franco, R. (1993). Evaluación de proyectos sociales. Madrid: Siglo Veintiuno.

De la Cruz Martín-Romo, C. (2003). Educación de las sexualidades: los puntos de partida de la Educación Sexual [Monografía]. Revista Española de Sexología, 119, 1-122.

Dezin, N. K., \& Lincoln, Y. S. (2000). Handbook of Qualitative Research. Thousand Oaks: Sage.

Farr, G., Gebelnick, H., Sturgen, K., \& Dorglinger, L. (1994). Contraceptive efficacy and acceptability of the female condom. American Journal of Public Health, 84, 19601964.

Feliz, T., \& Ricoy, M.C. (2003). El descubrimiento de la dimensión cualitativa a través de un foro educativo. En A. Medina, \& S. Castillo (Coords.), Metodología para la realización de proyectos de investigación y tesis doctorales (pp. 131-165). Madrid: Universitas.

Goetz, J., \& LeCompte M. (1998). Etnografía y diseñocualitativo en investigación educativa. Madrid: Morata. 
Gollub, E.L., Brown, E.L., Savouillan, M.,Waterlot, J., \& Coruble, G. (2002). A community-based safer-sex intervention for women: Results of a pilot study in south-eastern France. Culture Health \& Sexuality, 4, 21-41

Haignere, C. S., Gold, R., Maskovsky, J., Ambrosini, J., Rogers. C. L., \& Gollub, E. (2000). High-risk adolescents and female condoms: Knowledge, attitudes, and use patterns. The Journal of Adolescents Health, 26, 392- 398.

Hoke, T. H., Feldblum, P. J., Van Damme, K., Nasution, M. D., Grey, T. W., Wong, E. L.,E. Ralimamonjy, L., Raharimalala, L. \& Rasamindrakotroka, A. (2007). Temporal trends in sexually transmitted infection prevalence and condom use following introduction of the female condom to Madagascar sex. Internacional Journal of STD \& AIDS, 18, 461- 466.

Instituto de la Juventud (INJUVE) (2005). Condiciones de vida y situación de los jóvenes. Informe de juventud en España 2004. Ministerio de Trabajo y Asuntos Sociales. Recuperado de www.mtas.es

Instituto de Salud Carlos III (2005). Vigilancia epidemiológica del sida en España. Registro nacional de casos de sida. Informe semestral, 2. Recuperado de http://www.isciii. es/htdocs/centros/epidemiologia/epi_sida.jsp

Kulczycki, A., Kin, D. J., Duerr, A., Jamieson, D. J., \& Macaluso, M. (2004). The acceptability of the female and male condom: A randomized crossover trial. Perspectives on Sexual and Reproductive Health, 36, 114-119.

Lameiras, M., Faílde, J. M., Saco, A., \& Rodríguez, Y. (2006). A qualitative study of the viability of usage of the female condom among university students. International Journal of Clinical and Health Psychology, 6, 189-199.

Lameiras, M., Núñez, A.M., Rodríguez, Y., Breton, J., \& Agudelo, D. (2007). Conocimiento y viabilidad de uso del preservativo femenino em jóvenes universiatiros españoles. International Journal of Clinical and Health Psychology, 7, 9-25.
Latka, M. (2001). Female-initiated barrier methods for the prevention of STI/HIV: where are we now? Journal of Urban Health: Bulletin of the New York Academy of Medicine, 78, 571- 580.

Lawson, M.L., Macaluso, M., Duerr, A., Hortin, G., Hammond, K. R., Blackwell, R., Artz, L., \& Bloom, A. (2003). Partner characteristics, intensity of the intercourse, and semen exposure during use of the female condom. American Journal of Epidemiology, 157, 282288.

Mantel, J. E., Hoffman, S., Exner, T. M., Stein, Z. A., \& Atkins, K. (2003). Family planning providers' perspectives on dual protection. Perspectives on Sexual and Reproductive Health, 35, 71-78.

Marcelo, C. (1992). Dar sentido a los datos: la combinación de perspectivas cualitativas y cuantitativas en el análisis de entrevistas. En C. Marcelo (Coord.), La investigación sobre la formación del profesorado. Métodos de investigación y análisis de datos (pp. 1348). Buenos Aires (Argentina): Cincel.

Marshall, S., Giblin, P., Simpson, P., \& Backos, A. (2002). Adolescent girls' perception and experiences with the reality female condom. Journal of Adolescence Health, 31, 5-6.

Mcniff, J., Lomax, P., \& Whitehead, J. (2001). You and your action research project. London: Hyde Publications.

Neilands, T. B., \& Choi, K. H. (2002). A validation and reduced from of the female condom: Attitudes scales. AIDS Education and Prevention, 14, 158-174.

Núñez, A. M., (2006). Conducta sexual $y$ conocimiento y viabilidad de uso del preservativo femenino en jóvenes universitarios/as durante el período 20002005 (Tesis de licenciatura no publicada). Facultad de Ciencias de la Educación: Universidad de Vigo.

Okunlola, M. A., Morhason-Bello, I. O., Owonikoko, K. M., \& Adekunle, A. O. 
(2006). Female condom awareness, use and concerns among Nigerian female undergraduates. Journal of Obstetrics and Gynaecology: the Journal of Obstetrics and Gynaecology, 26, 353-356.

Onusida (2007). Informe sobre la epidemia mundial del SIDA. Recuperado de http://data.unaids.org/pub/ SPISlides/2007/2007_epiupdate_es.pdf

Pelto, P., \& Pelto, G. (1978). Anthropological research: The structure of inquiry. New York: Harcourt Brace.

Pérez Juste R. (2006). Evaluación de programas educativos. Madrid: La Muralla.

Programa de Naciones Unidas pra el Desarrollo (PNUD) (2006). Informe anual 2006: una alianza mundial para el desarrollo. Recuperado de http://www.undp.org/ spanish/publicaciones/informeanual2006/ IAR06_SP.pdf

Preusseler, G. M., Micheletti, V. C., \& Pedro, E. N. (2002). Preservativo feminino: uma possibilidade de autonomía para mulheres HIV positivas. Revista Brasileira de Enfermagen, 56, 699-701.

Prudhomne, M., Boucher, J., Delberghe, E., Christiman, H., \& Leroux, M.C. (2005). Female condom vs male condom: Ofter a choice. Gynécologie, Obstétrique \& Fertilité, 33 (11), 891-897.

Robinson, B., Uhl, G., Miner, M., Bockting, W., Schelteme, K. E., Rosser, B. R. S., \& Westover, B. (2002). Evaluation of sexual health approach prevent HIV among low, urban primarily African American women: Results of a randomized controlled trial. AIDSEducation and Prevention, 14, 81-96.

Smith, J. B., Nkhama, G., \& Trottier, D. A. (2001). Female condom reuse in Lusaka, Zambia: evidence from 12 cases. Journal of Urban Health: Bulletein of The New York Academy of Medicine, 78, $638-646$.
Spizzichino, L., Pedone, G., Gattari, P., Gallo, P., Valli, R., \& Rezza, G. (2007). The female condom: Knowledge, attitute, and willingness to use, the first Italian study. Annali Dell'istituto Superiore Di Sanitá, 43, 419-424.

Trilla, J. (1993). Otras educaciones: animación sociocultural, formación de adultos y ciudad educativa. Barcelona: Anthropos.

Walker, R. (1989). Métodos de investigación para el profesorado. Madrid: Morata.

Welbourn, A. (2006). Sex, life, and female condom: Some Views of HIV positive women. Reproductive Health Matters, 14, 32- 40. 
